\title{
LES/CMC Modelling of Ignition and Flame Propagation in a Non-premixed Methane Jet
}

\author{
Huangwei Zhang ${ }^{1}$, Andrea Giusti ${ }^{2}$ and Epaminondas Mastorakos ${ }^{2}$ \\ ${ }^{1}$ Department of Mechanical Engineering, National University of Singapore, 9 Engineering Drive 1, \\ Singapore 117576, Republic of Singapore \\ ${ }^{2}$ Department of Engineering, University of Cambridge, Cambridge CB2 1PZ, United Kingdom
}

Corresponding author: Huangwei Zhang

Assistant Professor

Department of Mechanical Engineering

Faculty of Engineering, National University of Singapore

9 Engineering Drive 1, Singapore 117576

Republic of Singapore

Tel: +44 6516 2557, Fax: +44 67791459

Email: mpezhu@nus.edu.sg

\section{Colloquium topic: 5. Turbulent flames}

\section{Alternative Colloquium topic: N/A}

\section{Paper length (method 1):}

Main Text:

Equations:

References:

Figure 1:

Figure 2:

Figure 3:

Figure 4:

Figure 5:

Figure 6:

Figure 7:

Figure 8:

Figure 9: word processor count

$(5$ lines +6 blank lines $) \times(7.6$ words $/$ line $) \times(1$ column $)$

$(31+2) \times(2.3$ lines $/$ reference $) \times(7.6$ words $/$ line $)$

$(45 \mathrm{~mm}+10) \times(2.2$ words $/ \mathrm{mm}) \times(1$ column $)+(17$ words in caption $)$

$(48 \mathrm{~mm}+10) \times(2.2$ words $/ \mathrm{mm}) \times(1$ column $)+(20$ words in caption $)$

$(48 \mathrm{~mm}+10) \times(2.2$ words $/ \mathrm{mm}) \times(1$ column $)+(29$ words in caption $)$

$(70 \mathrm{~mm}+10) \times(2.2$ words $/ \mathrm{mm}) \times(1$ column $)+(30$ words in caption $)$

$(142 \mathrm{~mm}+10) \times(2.2$ words $/ \mathrm{mm}) \times(1$ column $)+(53$ words in caption $)$

$(165 \mathrm{~mm}+10) \times(2.2$ words $/ \mathrm{mm}) \times(1$ column $)+(56$ words in caption $)$

$(35 \mathrm{~mm}+10) \times(2.2$ words $/ \mathrm{mm}) \times(1$ column $)+(25$ words in caption $)$

$(55 \mathrm{~mm}+10) \times(2.2$ words $/ \mathrm{mm}) \times(1$ column $)+(29$ words in caption $)$

$(72 \mathrm{~mm}+10) \times(2.2$ words $/ \mathrm{mm}) \times(1$ column $)+(65$ words in caption $)$

$$
\begin{array}{ll}
= & 3580 \\
= & 60 \\
= & 576 \\
= & 116 \\
= & 125 \\
= & 156 \\
= & 206 \\
= & 389 \\
= & 441 \\
= & 124 \\
= & 172 \\
= & 245
\end{array}
$$

Total $=6190$ words

\section{Supplemental Material: none}

Color reproduction: no (color figures are to be printed in gray scale) 


\title{
LES/CMC Modelling of Ignition and Flame Propagation in a Non-premixed Methane Jet
}

\author{
Huangwei Zhang ${ }^{1 *}$, Andrea Giusti ${ }^{2}$ and Epaminondas Mastorakos ${ }^{2}$ \\ ${ }^{1}$ Department of Mechanical Engineering, National University of Singapore, 9 Engineering Drive 1, \\ Singapore 117576, Republic of Singapore \\ ${ }^{2}$ Department of Engineering, University of Cambridge, Cambridge CB2 1PZ, United Kingdom
}

\begin{abstract}
The Large Eddy Simulation (LES) / Conditional Moment Closure (CMC) model with detailed chemistry is used for modelling spark ignition and flame propagation in a turbulent methane jet in ambient air. Two centerline and one off-axis ignition locations are simulated. We focus on predicting the flame kernel formation, flame edge propagation and stabilization. The current LES/CMC computations capture the three stages reasonably well compared to available experimental data. Regarding the formation of flame kernel, it is found that the convection dominates the propagation of its downstream edge. The simulated initial downstream and radial flame propagation compare well with OH-PLIF images from the experiment. Additionally, when the spark is deposited at off-centerline locations, the flame first propagates downstream and then back upstream from the other side of the stoichiometric isosurface. At the leading edge location, the chemical source term is larger than others in magnitude, indicating its role in the flame propagation. The time evolution of flame edge position and the final liftoff height are compared with measurements and generally good agreement is observed. The conditional quantities at the stabilization point reflect a balance between chemistry and micro-mixing. This investigation, which focused on model validation for various stages of spark ignition of a turbulent lifted jet flame through comparison with measurements, demonstrates that turbulent edge flame propagation in
\end{abstract}

* Corresponding author. E-mail: mpezhu@nus.edu.sg. Tel: +65 65162557. 
non-premixed systems can be reasonably well captured by LES/CMC.

Keywords: Flame kernel formation, flame edge propagation, flame stabilization, large eddy simulation, conditional moment closure 


\section{Introduction}

Spark ignition of a flammable mixture is a fundamental problem in combustion science $[1,2]$. It deals with a transient process, from flame kernel formation, to subsequent expansion and to fully reacting state. Turbulent flame ignition is not only influenced by the canonical quantities such as minimum ignition power and critical flame radius, but it is also strongly dependent on the local turbulence characteristics, fuel properties and also burner configurations [1, 2]. Therefore, understanding of the ignition of turbulent flames is still a challenging task for both experimentalists and modellers.

Because of its simple configuration, ignition of jet flows has been experimentally investigated in terms of ignition transients, ignition probability, flame propagation and stabilization. For instance, the correlations between jet/co-flow parameters and the critical streamwise locations were investigated in turbulent lifted methane flames [3]. More detailed measurements were also made for diluted methane jet flows by Ahmed and Mastorakos [4], who focused on three stages of flame evolution when a spark is applied, i.e. flame kernel growth, downstream flame propagation and radial expansion, and upstream flame propagation. This latter phase relies on edge flame propagation $[1,5]$. The ignition probability was also discussed for different operating conditions, which provides further insights about ignitability of jet flows compared to the earlier work $[6,7]$.

The jet ignition experiments in Ref. [4] have been used to examine flame ignition models. Reynolds-Averaged Navier-Stokes (RANS) simulations with CMC were applied by Richardson and a significant difference was observed between the simulated and measured flame front location [8]. Recently, a series of LES studies for this flame were reported [9-11], demonstrating the accuracy of their respective sub-grid scale combustion models in predicting transient flame evolution. LES/CMC has been applied for simulation of spark ignition of a non-premixed bluff-body flow [12], in which the recirculating flame is compact and the transient is relatively short [13]. More recently, Rosiak and Tyliszczak simulated the flame development and propagation after spark ignition of a turbulent non- 
premixed hydrogen jet in oxy-combustion regimes with LES/CMC [14]. However, in this work, no comparisons with measurements were made and therefore the accuracy of the results cannot be assessed.

This paper will discuss LES/CMC simulations of development and stabilization of a nonpremixed jet flame following localized spark ignition, and hence test the second and third phases of overall burner ignition, as classified by Mastorakos [1]. In particular, in the ignition of a non-premixed jet, and depending on the spark location, flame expansion spans a range of modes, from stratified, to “premixed/non-premixed" or "partially premixed", to triple and edge flames. The LES/CMC model has not been tested explicitly for such flames with mixed modes, but they are at the heart of the practical ignition processes. Getting the lift-off height right (partially achieved before with RANS/CMC like in Ref. [15] or LES/CMC like in Ref. [16]) tests only the last phase. Note that in Ref. [16], a lifted nonpremixed flame produced by a fuel jet issuing into a hot vitiated co-flow was studied. To the authors' knowledge, this is the first study where the accuracy of LES/CMC in capturing flame propagation across a wide range of flame regimes after spark ignition is assessed through quantitative comparisons with the experimental data. In the following, models and flame information will be presented in Section 2, while the results and discussion will be given in Section 3, followed by the conclusions in the last Section.

\section{Model and flame information}

\subsection{LES and CMC models}

The LES equations for mass, momentum, and mixture fraction are derived by applying low-pass Favre filtering to their respective instantaneous equations. The sub-grid scale stress tensor is closed by

the constant Smagorinsky model. The mixture fraction sub-grid variance $\widetilde{\xi^{\prime \prime 2}}$ is modeled by $\widetilde{\xi^{\prime \prime 2}}=$ $c_{v} \Delta^{2} \nabla \tilde{\xi} \cdot \nabla \tilde{\xi}$ with $C_{V}=0.1$ [17] and $\Delta$ the filter width, estimated as the cube root of the LES cell volume.

The filtered scalar dissipation rate $\widetilde{N}$ includes the resolved and sub-grid parts [18]

$$
\widetilde{N}=\widetilde{N}_{r e s}+\widetilde{N}_{s g s}
$$


in which $\widetilde{N}_{r e s}=D \nabla \tilde{\xi} \cdot \nabla \tilde{\xi}$ and $\widetilde{N}_{s g s}=\frac{c_{N}}{2} \frac{\mu_{t}}{\bar{\rho} \Delta^{2}} \widetilde{\xi^{\prime \prime 2}}$ with $C_{N}=42$ [19]. $D$ is the molecular diffusivity, $\mu_{t}$ is the turbulent viscosity and $\bar{\rho}$ is the filtered density.

The integral form of the CMC governing equations for the conditionally filtered mass fraction of $\alpha$ th species, i.e. $Q_{\alpha} \equiv \widetilde{Y_{\alpha} \mid \eta}$, reads $[20,21]$

$$
\begin{aligned}
& \underbrace{\int_{\Omega^{\mathrm{CMC}}} \frac{\partial Q_{\alpha}}{\partial \mathrm{t}} \mathrm{d} \Omega}_{\mathrm{T}_{0}}+\underbrace{\int_{\Omega^{\mathrm{CMC}}} \nabla \cdot\left(\widetilde{\mathrm{U}} \eta Q_{\alpha}\right) \mathrm{d} \Omega}_{\mathrm{T}_{1}}= \\
& \underbrace{\int_{\Omega_{\mathrm{CMC}}} Q_{\alpha} \nabla \cdot \widetilde{\mathrm{U} \mid \eta} \mathrm{d} \Omega}_{\mathrm{T}_{2}}+\underbrace{\int_{\Omega^{\mathrm{CMC}}} \widetilde{N \mid} \eta \frac{\partial^{2} Q_{\alpha}}{\partial^{2} \eta} \mathrm{d} \Omega}_{\mathrm{T}_{3}}+\underbrace{\int_{\Omega^{\mathrm{CMC}}} \widetilde{\omega_{\alpha} \mid \eta} \mathrm{d} \Omega}_{\mathrm{T}_{4}}+\underbrace{\int_{\Omega^{\mathrm{CMC}}} \nabla \cdot\left(D_{t} \nabla Q_{\alpha}\right) \mathrm{d} \Omega}_{\mathrm{T}_{5}},
\end{aligned}
$$

where $t$ is time and $\eta$ is the sample space variable for $\xi . \Omega^{\mathrm{CMC}}$ denotes the CMC cell. $\widetilde{U} \eta \eta$, $\widetilde{N \mid \eta}$, and $\widetilde{\omega_{\alpha} \mid \eta}$ are the conditionally filtered velocity, scalar dissipation rate, and reaction rates of the $\alpha$-th species, respectively. $D_{t}$ is the sub-grid scale diffusivity, given by $D_{t}=\mu_{t} / \bar{\rho} S c_{t}$ with turbulent $\mathrm{Schmidt}$ number $S c_{t}=0.4[22]$.

The assumption $\widetilde{\mathbf{U} \eta \eta} \approx \widetilde{\mathbf{U}}$ is adopted for Eq. (2). For $\widetilde{N \mid \eta}$, the Amplitude Mapping Closure (AMC) model [23] is applied, i.e. $\widetilde{N \mid \eta}=N_{0} G(\eta)$, where $\quad N_{0}=\widetilde{N} / \int_{0}^{1} \tilde{P}(\eta) G(\eta) d \eta$ and $G(\eta)=$ $\exp \left(-2\left[\operatorname{erf}^{-1}(2 \eta-1)\right]^{2}\right)$. The filtered scalar dissipation rate $\widetilde{N}$ is calculated with Eq. (1). $\tilde{P}(\eta)$ is the Filtered probability Density Function (FDF) and is estimated with beta-function. The first order CMC model is used, so that $\widetilde{\omega_{\alpha} \mid \eta} \approx \omega_{\alpha}\left(Q_{1}, \ldots Q_{n}, Q_{T}\right)$. Here $n$ is the number of species and $Q_{T} \equiv \widetilde{T \mid \eta}$ represents the conditionally filtered temperature. The filtered variable $\tilde{f}$ is calculated from the conditional value $\widetilde{f \mid \eta}$ through $\tilde{f}=\int_{0}^{1} \widetilde{f \mid \eta} \tilde{P}(\eta) d \eta$.

\subsection{Flame information and numerical implementation}

Fuel is injected through a circular pipe of diameter $D_{j}=0.005 \mathrm{~m}$ with bulk velocity equal to $U_{j}=$ $25.5 \mathrm{~m} / \mathrm{s}$. The fuel is air-diluted methane $\left(70 \% \mathrm{CH}_{4}\right.$ and $30 \%$ air by vol.). The stoichiometric mixture 
fraction $\xi_{s t}$ is 0.0976 , while the mixture fractions corresponding to rich and lean flammability limits are $\xi_{\text {rich }}=0.1582$ and $\xi_{\text {lean }}=0.0503$, respectively. Three different spark locations are selected, two centerline locations, i.e. $40 D_{j}$ and $30 D_{j}$, and one off-axis location with streamwise distance being $4 D_{j}$ and radial distance being $D_{j}$. For brevity, these three cases will be respectively termed as $40 D_{j}, 30 D_{j}$ and $4 D_{j}$ hereafter. The full flame development is simulated in both $40 D_{j}$ and $4 D_{j}$ cases. In addition, $30 D_{j}$ case is computed for investigating flame kernel formation and initial propagation.

The cylindrical LES domain starts at the jet exit with extension $170 D_{j} \times 75 D_{j} \times 2 \pi$ in the longitudinal, radial and azimuthal directions, respectively. The coordinate origin lies at the center of the jet exit. $x$ is the axial coordinate while $y$ and $z$ are the spanwise ones. A LES mesh of approximately $10,000,000$ tetrahedral cells with local refinement in the jet flame region is used. The domain for CMC in physical space is identical to the LES one with around 160,000 polyhedral CMC cells. The mixture fraction space is discretized by 51 nodes.

For the LES, the synthesized turbulent inlet method is used for inlet turbulence [24]. At the side and outflow boundaries, zero gradient condition for the velocities is assumed. Zero pressure gradient is enforced for the fuel inlet. The mixture fraction is unity at the central fuel jet exit, while zero gradient condition is assumed for the lateral and outflow boundaries. For the CMC domain, inert mixing solution is assumed at all the inlets. For the lateral and outlet boundaries, zero gradient conditions for $Q_{\alpha}$ are applied.

In mixture fraction space, at $\eta=0$ (oxidizer), the mass fractions of $\mathrm{O}_{2}$ and $\mathrm{N}_{2}$ are $23.3 \%$ and $76.7 \%$, respectively, while at $\eta=1$ (fuel), the mass fractions of $\mathrm{O}_{2}, \mathrm{~N}_{2}$ and $\mathrm{CH}_{4}$ are $10 \%, 32.9 \%$ and $57.1 \%$, respectively. The temperature for both boundaries is $298 \mathrm{~K}$. The initial CMC solution is assumed to be chemically inert, except the spark locations. The spark is numerically mimicked with a localized fully burning $Q_{\alpha}$ solution, obtained using a stand-alone 0D-CMC (Eq. 2 without $T 1, T 2$ and $T 5$ ) solver with the above boundary conditions and constant peak scalar dissipation rate $N_{0}=1001 / \mathrm{s}$. This strategy has 
also been used in previous studies [12]. In this work the spark radius is assumed to be $0.003 \mathrm{~m}$, resulting in approximately $100 \mathrm{CMC}$ cells in the numerical spark region. The burning flamelet is deposited at $t=$ $0 \mathrm{~s}$.

The finite volume CMC solver solving Eq. (2) is interfaced with the OpenFOAM ${ }^{\circledR}$ LES solver [25]. For LES, the numerical setup is identical to the one used in the previous work $[21,26,27]$. The time step is $4 \times 10^{-6} \mathrm{~s}$. The operator splitting method is applied for the solution of the discretized CMC equations, Eq. (2). First-order Euler time scheme is used for the transport in physical space (T1, T2 and T5). The first order upwind and second order central differencing schemes are used for the terms $T 1$ and T5. TDMA (Tri-Diagonal Matrix Algorithm) is applied in $\eta$-space for solving $T 3$, and the stiff ODE solver VODPK [28] is used for T4. The ARM2 mechanism (19 species and 15 reactions) [29] is used. For $t<0.0001 \mathrm{~s}$, relaxation between time steps for the density in LES solver is applied to avoid numerical stability issues. Bi-directional data exchange between two solvers is executed for each time step, following the strategy detailed in Refs. $[20,26] .180$ processors on the Cirrus cluster of the UK National HPC Facilities are used for the current simulations and $0.01 \mathrm{~s}$ of physical time can be obtained for 24 hours wall clock time.

Mean and rms values of filtered velocity fields from LES of an air jet with the above LES mesh and $U_{j}=21 \mathrm{~m} / \mathrm{s}$ were compared with the measurements from Ref. [4] and good agreement was found. Furthermore, the mean of filtered mixture fraction field for the non-reacting air-diluted methane $\left(\mathrm{CH}_{4}\right.$ : air=70\%:30\% by vol.) jet flow with $U_{j}=25.5 \mathrm{~m} / \mathrm{s}$, which will be used as the initial fields for the following ignition studies, is also well predicted compared to the correlations by Richards and Pitts [30]. These validations will not be expanded here due to the space limitation.

\section{Results and discussion}

\subsection{Flame kernel formation and initial flame expansion}


Figure 1 shows the centerline profiles of flammability factor, calculated from LES of non-reacting air-diluted methane jet with $U_{j}=25.5 \mathrm{~m} / \mathrm{s}$. The mean mixture fraction is also added. The rich and lean flammability limits lie at approximately $x=33 D_{j}$ and $80 D_{j}$, respectively. The flammability factor $F$ is estimated from $F=\int_{\xi_{\text {lean }}}^{\xi_{\text {rich }}} P(\eta) d \eta[28]$, in which $P(\eta)$ is the beta-shaped PDF calculated with the mean mixture fraction and its standard deviation. $F$ increases until $x=45 D_{j}$ (roughly corresponding to locations of $\xi_{s t}$ ), and then gradually decreases and after $x=110 D_{j}$ it tends to zero. Finite $F$ also exists in the regions beyond the flammability limits. These trends were also observed by Smith et al. in the ignition experiments in jet flows with different fuels [31]. It was shown from their experiments that, along the jet centerline, the flammability factor $F$ is a good approximation to the probability of flame kernel formation, which is not calculated in the present work due to computational cost. As shown in Fig. 1, $F$ for spark locations at $x=30 D_{j}$ and $40 D_{j}$ is respectively 0.4 and 0.9 . Indeed, in the computational ignition attempts (25 various initial fields were chosen) with different initial fields for both locations, failure of flame kernel formation at $x=30 D_{j}$ is more frequent than that at $x=40 D_{j}$.

The movement of the newly generated flame kernel is a significant aspect for this ignition experiment [4], and also a significant measure for model validation. To clarify this, the successfully initiated flame kernel at $t=0.01 \mathrm{~s}$, after the spark is deposited at $t=0 \mathrm{~s}$ and $x=40 D_{j}$, is visualized through the resolved $\mathrm{OH}$ mass fraction $\tilde{Y}_{O H}$ in Fig. 2(a). The shape is still almost circular, although the front is slightly distorted by the local turbulence. The highest concentration of $\mathrm{OH}$ is not close to the $\xi_{s t}$ isolines; instead, the high $\tilde{Y}_{O H}$ lies near the interface between the flame kernel and fresh mixture. This can be

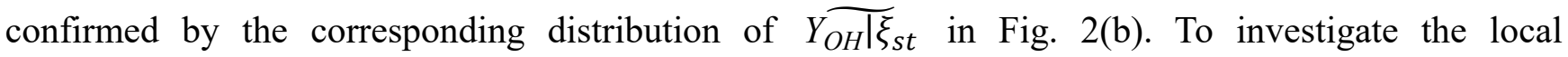
contributions of different physics indicated by the CMC equation, Eq. (2), budget analysis for $\widetilde{Y_{O H} \mid \eta}$ is shown in Fig. 3. Two locations along the centerline are selected, i.e. $Q 1\left(x=50 D_{j}\right)$ and $Q 2\left(x=40 D_{j}\right)$, roughly corresponding to the upstream and downstream edges of the considered kernel. For both $Q 1$ and 
$Q 2$, the scalar dissipation rate (T3) and sub-filter diffusion (T5) terms are negligible, while the conditional dilatation term, $T 2$, only shows weak contribution. For $Q 1$, both conditional convection and chemistry are sink terms for $0.05<\eta<0.125$, while for $\eta \approx 0.135$ chemistry dominates over the convection and becomes source term. For $Q 2$, chemistry is balanced by convection for most locations (e.g. $0.08<\eta<$ $0.1)$ in $\eta$-space. Therefore, the downstream expansion of the kernel is dominated by the transport in physical space.

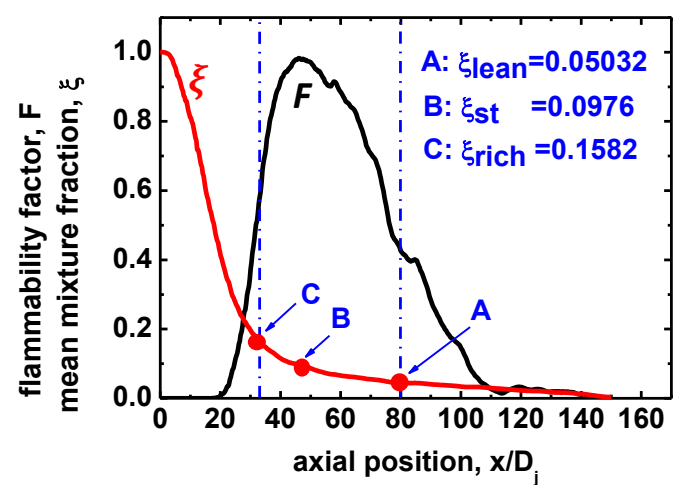

Fig. 1 Centerline distributions of mean mixture fraction and flammability factor. Dashed vertical lines: rich and lean flammability limits.

The evolution of flame after the kernel is formed is plotted in Fig. 4, and the comparison is made between OH-PLIF [4] and $\tilde{Y}_{O H}$ from the LES/CMC. At $t=0.005 \mathrm{~s}$, the computational results show that the flame first expands downstream. At $t=0.01 \mathrm{~s}$, downstream propagation occurs, accompanied by a radial expansion of the flame. $0.02 \mathrm{~s}$ later (see Fig. 4c), the flame dimension is further increased. The computed radial width is slightly over $8 D_{j}$, close to the measured value $7 D_{j}[4]$. Nevertheless, differences also exist. For instance, in the experiments the flame starts to propagate upstream at about $0.017 \mathrm{~s}$ [4] and at $t=0.03 \mathrm{~s}$ in Fig. 4(c) the flame has already shown significant upstream movement. In the simulation results, the flame does not move upstream considerably at this instant. This can be justified by the budget analysis in Fig. 3(b), i.e. the balance between convection and chemistry in $\eta$-space. 


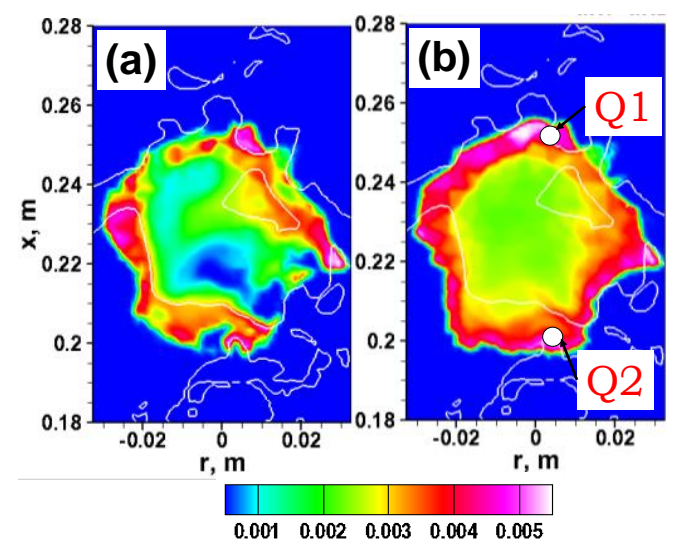

Fig. 2 Contours of (a) $\tilde{Y}_{O H}$ and (b) ${\widetilde{Y_{O H} \mid \xi_{s t}}}_{\text {. }}$. Results from $40 D_{j}$ case. White iso-lines: $\xi_{s t}=$ 0.0976. $t=0.01 \mathrm{~s}$.

(a) $\mathrm{t}=5 \mathrm{~ms}$
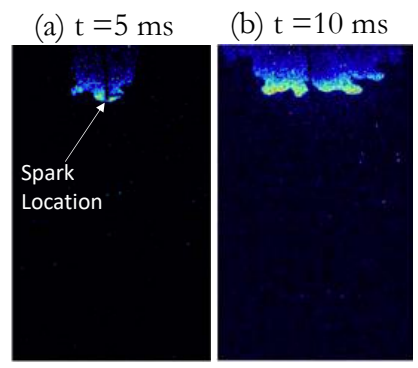

(c) $\mathrm{t}=30 \mathrm{~ms}$
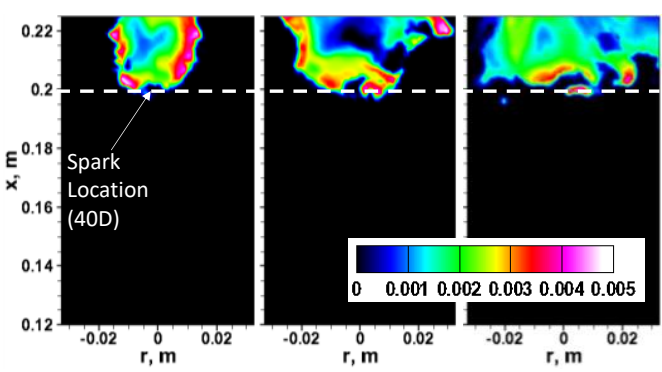

Fig. 4 Comparisons of the time evolution of flame position for $40 D_{j}$ case. First row: OH-PLIF [4]; second row: $\tilde{Y}_{O H}$ from LES/CMC. Image size: $21 D_{j} \times 13 D_{j}$; bottom location: $24 D_{j}$ off the jet exit.

\subsection{Edge flame propagation and stabilization}


The time evolution of filtered temperature $\tilde{T}$ from off-axis ignition $4 D_{j}$ case is plotted in Fig. 5 . After $t$ $=0.005 \mathrm{~s}$, the flame kernel has formed, but the hot pocket is still confined close to the spark location as shown in Fig. 5(a) and the inset for $x=4 D_{j}$. Then the flame starts to propagate downstream along the $\xi_{s t}$ isolines and, at $t=0.029 \mathrm{~s}$, it has reached $20 D_{j}$ as shown in Fig. $5(\mathrm{~b})$. This is close to the distance $\left(23 D_{j}\right)$ observed from high-speed camera images for this case (see Fig. 9 in Ref. [4]). The flame also expands circumferentially, towards both sides of $\xi_{s t}$ iso-lines, which is noticeably shown in the two insets in Fig. 5(b). Note that the circumferential propagation along $\xi_{s t}$ at $x=7.5 D_{j}$ seems faster than at $x=5 D_{j}$. Approximately $0.01 \mathrm{~s}$ later in Fig. $5(\mathrm{c})$, for the location of $20 D_{j}, \tilde{T}$ has increased for the whole isolines. However, the right branch below $15 D_{j}$ is still not ignited. Then at $t=0.049 \mathrm{~s}$ in Fig. $5(\mathrm{~d})$, downstream movement of the flame along both $\xi_{s t}$ branches is observable. The flame along the right branch starts to propagate upstream towards the jet exit. The hot gas is transported further and at $t=0.14 \mathrm{~s}$ the flame is fully developed as in Fig. 5(f). The streamwise stabilization point (i.e. lift-off height) is around $10 D_{j}$, close to the measured results (see Fig. 9 in Ref. [4]). The transient ignition process with off-centerline spark is different from that with centerline cases which will be discussed later. The flame leading edge generally moves from spark towards jet exit as observed from measurements [4], although the leading point varies spatially with the flame development duration [9].

To understand the mechanism of edge flame propagation, it is relevant to analyze the evolution of flame structures in $\eta$-space (here visualized with $\widehat{Y_{O H} \mid \xi_{s t}}$ ). ${\widetilde{Y_{O H} \mid}}_{s t}$ at three different instants during the flame propagation (before reaching the stabilization point) is shown in Figs. 6(a)-6(c) for the $30 D_{j}$ ignition case. The corresponding $\tilde{Y}_{O H}$ for the same instants is also shown in Figs. 6(d)-6(f). At $t=0.005$ s, burning flame structure with high $\widehat{Y_{O H} \mid \xi_{s t}}$ is very localized, around the spark location. This is similar to the flame kernel from the $40 D_{j}$ case in Fig. 2(b). When $t=0.05 \mathrm{~s}$, the burning flame structure has expanded downstream and radially, beyond the $\xi_{s t}$ isolines for $x>10 D_{j}$. This leads to finite $\tilde{Y}_{O H}$ in physical space $\left(x>10 D_{j}\right)$ as shown in Fig. 6(e). At $t=0.23 \mathrm{~s}$, the region with high $\widetilde{Y_{O H} \mid \xi_{s t}}$ is further 

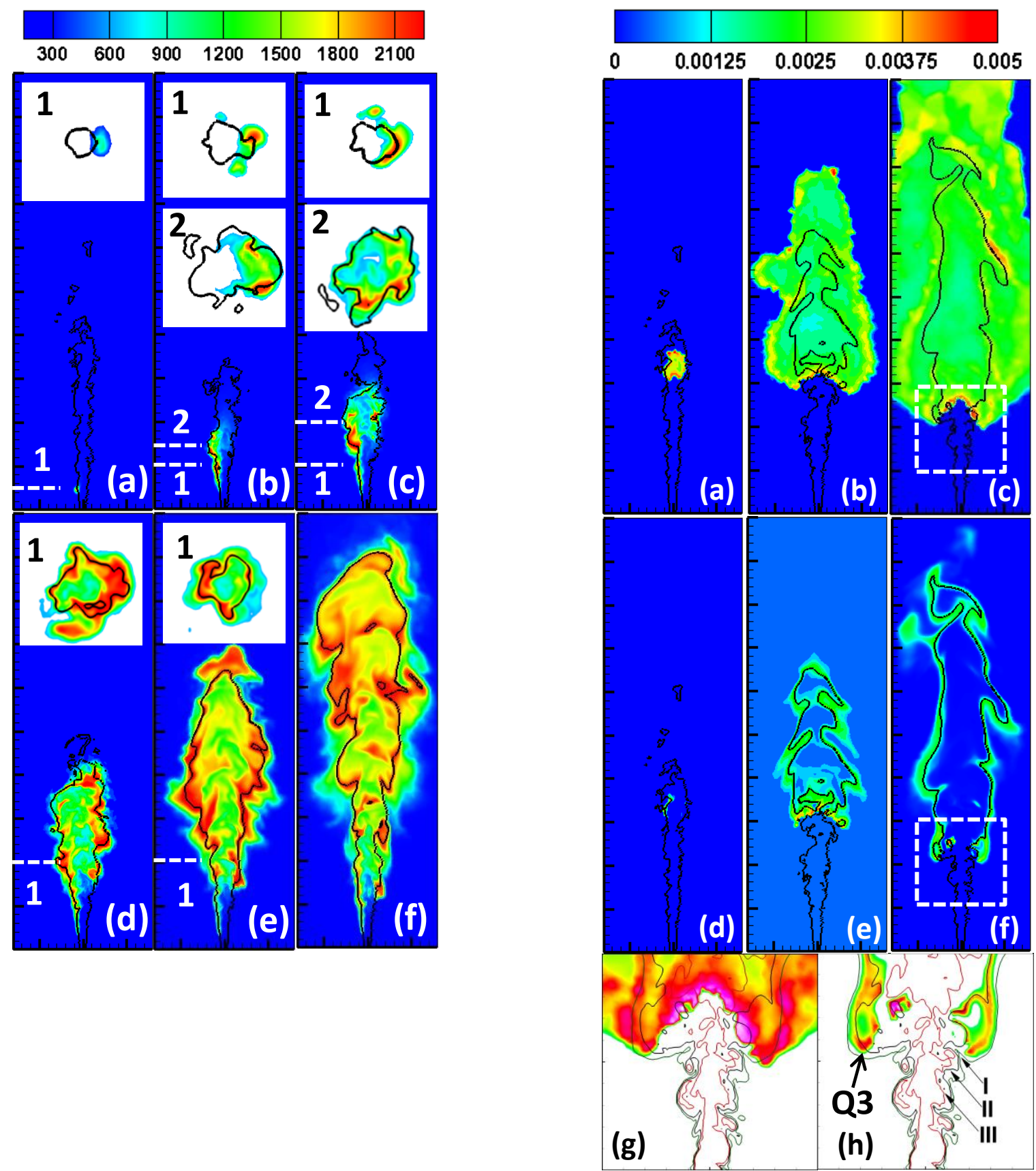

Fig. 5 Time evolutions of $\tilde{T}$ (in K) for off-axis ignition $4 D_{j}$ at $t=$ (a) $0.005 \mathrm{~s}$, (b) $0.029 \mathrm{~s}$, (c) 0.038 $\mathrm{s}$, (d) $0.049 \mathrm{~s}$, (e) $0.08 \mathrm{~s}$ and (f) $0.14 \mathrm{~s}$. Iso-lines: $\xi_{s t}$. Interval between major ticks is $10 D_{j}$. Insets are the top-view at the specified streamwise locations marked with dashed lines.

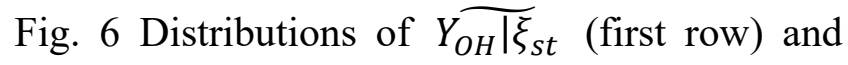
$\tilde{Y}_{O H}$ (second row) for $30 D_{j}$ case at $t=(\mathrm{a}, \mathrm{d}) 0.005$ s, (b,e) $0.05 \mathrm{~s}$, and (c,f) 0.23 s. Iso-lines: $\xi_{s t}$. Interval between major ticks in (a)-(f) is $10 D_{j} .(\mathrm{g})$ and (h) correspond to dashed boxes in (c) and (f), respectively. I, II, III are $\xi_{\text {lean }}, \xi_{\text {st }}$ and $\xi_{\text {rich }}$ isolines. 
increased. The structure of the leading edge in Figs. 6(c) and 6(f) is enlarged with $\xi_{\text {rich, }} \xi_{\text {st }}$ and $\xi_{\text {lean }}$ overlaid in Figs. 6(g) and 6(h). Generally, high $\tilde{Y}_{O H}$ is confined within $\xi_{\text {lean }}$ isolines, and mostly follows the $\xi_{\text {st }}$ isolines.

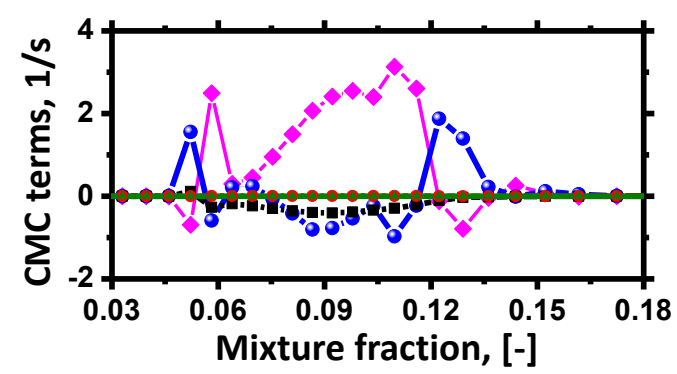

Fig. 7 Budget analysis of individual terms in Eq. (2) for $\widetilde{Y_{O H} \mid \eta}$ at flame leading edge (marked as $Q 3$ in Fig. 6h). Legend as in Fig. 3.

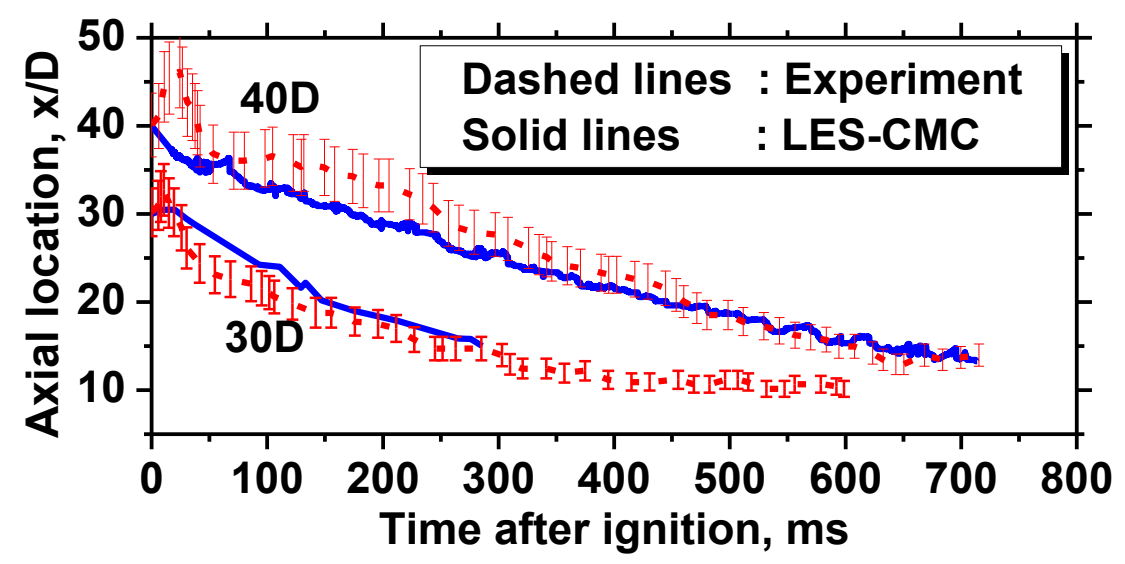

Fig. 8 Flame edge position versus time for $30 D_{j}$ and $40 D_{j}$ cases. Experimental data from Ref. [4]. Error bars represent $9 \%$ of the averaged flame edge positions reported in Ref. [4].

Similar to Fig. 3, budget analysis of one CMC cell (marked as $Q 3$ in Fig. 6h) is made and the contributions of each term in Eq. (2) for $\widetilde{Y_{O H} \mid \eta}$ are plotted in Fig. 7. For this $Q 3$ cell, the magnitudes of T3 and T4 are much larger than others. For most of the shown range of mixture fraction, T4 term (conditional chemical source term) acts as a source term, dominating the net contributions to local $\overline{Y_{O H} \mid \eta}$. For $0.06<\eta<0.12, T 3$ (micro-mixing) and $T 1$ (convection) are weak sink terms. Note that here $T 1$ is a 
net contribution of numerical fluxes from all the neighboring $\mathrm{CMC}$ cells, including influx and efflux. The flame edge movement towards the jet exit is accompanied by propagation of conditional burning flame structures in physical space as shown in Figs. 6(c) and 6(f). This propagation is expected to be affected by flow transport ( $T 1$ and $T 5$ ), although their contribution in Fig. 7 is small, which would be dominated again by the chemistry term $T 4$ at new flame edge locations.

The time evolution of the flame edge position for $30 D_{j}$ and $40 D_{j}$ cases predicted from LES/CMC is shown in Fig. 8. Results from a single ignition simulations per spark location are considered, while the experimental data are averaged based on ten independent measurements [4]. The flame edge position is estimated as the longitudinally lowest location where $\tilde{Y}_{O H}$ is below the threshold value 0.0005 . For the $40 D_{j}$ case, the flame edge moves upstream gradually, which is correctly captured for most of the shown time. Meanwhile, at about $t=0.7 \mathrm{~s}$, the flame stabilizes at around $15 D_{j}$, consistent with the experiemntal data in Fig. 8. Two differences should be discussed. Firstly, the LES/CMC does not reproduce the flame kernel downstream propagation for the initial 0.03-0.04 s; instead, the flame edge position monotonically decreases after the spark is deposited. Secondly, for $0 \leq t \leq 0.45 \mathrm{~s}$, the flame propagates faster than the measured results, leading to lower edge position (although still close to the lower limit of the error bar) at the same instant. These may be attributed to the randomly selected initial non-reacting mixing fields, or may be caused by the over-prediction of the reactivity along the leading edge by the CMC model, as discussed in Figs. 5 and 6 . Regarding the $30 D_{j}$ case, only the early propagation stage $(t \leq 0.28 \mathrm{~s})$ is simulated, and agreement between LES/CMC results and measurements is also satisfactorily good. The computed flame edge position starts to be close to the measurement at around $t=0.15 \mathrm{~s}$. 


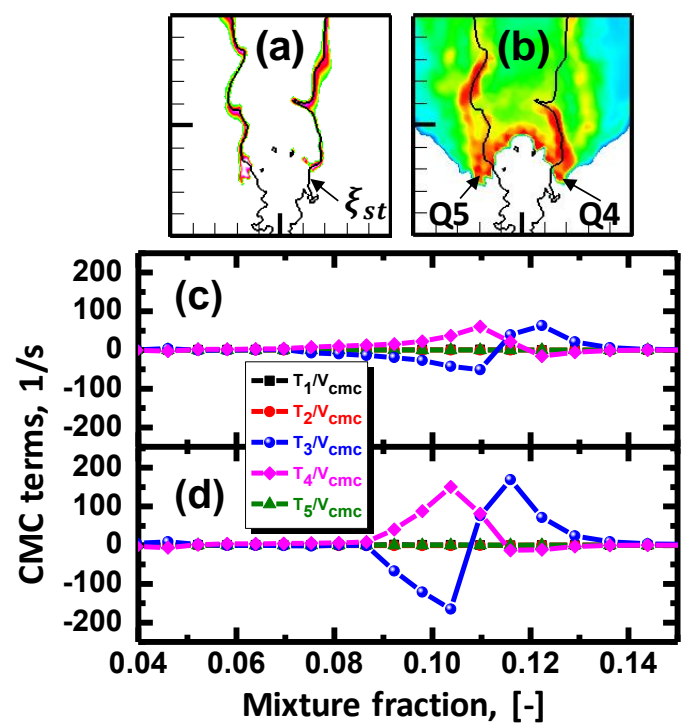

Fig. 9 Contours of (a) $\tilde{Y}_{O H}$ and (b) $\widehat{Y}_{O H} \mid \xi_{s t}$ for $40 D_{j}$ case at stabilization point $(t=0.71 \mathrm{~s})$. Budget analysis of individual terms in Eq. (2) for $\widetilde{Y_{O H} \mid \eta}$ for CMC cells (c) $Q 4$ and (d) $Q 5$ at flame leading edge. Iso-lines in (a) and (b): $\xi_{s t}$. Interval between major ticks in (a) and (b) is $10 D_{j}$ and the lowest shown height is $10 D_{j}$. Contour colors in (a) and (b): blue: 0; red: 0.005 .

Figures 9(a) and 9(b) show the contours of $\tilde{Y}_{O H}$ and $\widehat{Y O H}_{O H}$ for $40 D_{j}$ case when the jet flame stabilizes around $x=15 D_{j}$, as shown in Fig. 8 . Note that the results in Fig. 7 are from $30 D_{j}$ case. Through Figs. 7 and 9 we do not try to discuss the effects of different ignition locations on flame stabilization. Nevertheless, they are used to analyse flame behaviors in different stages, i.e. edge flame propagation

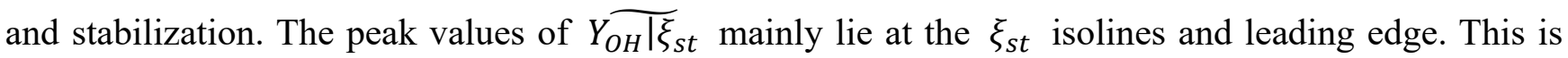
similar to the distributions demonstrated in Fig. 6. The budget analysis of the CMC equations for $\overline{Y_{O H} \eta \eta}$ is performed for left and right leading edge branches, i.e. $Q 4$ and $Q 5$ in Fig. 9(b), and the corresponding results are shown in Figs. 9(c) and 9(d). In both situations, the magnitude of chemistry term (T4) and micro-mixing term (T3) is much higher than others terms. Also, the conditional flame structure demonstrates the balancing or comparability between the chemical reaction and micro-mixing for 0.085 $<\eta<0.11$, which directly leads to the small change of $\widetilde{Y_{O H} \mid \eta}$ with respect to time in the local CMC 
cells. This is consistent with the previous CMC modelling of lifted jet flames [15]. Considering the CMC budget analysis for flame kernel in Fig. 3, flame edge propagation in Fig. 7 and flame stabilization in Fig. 9 together, one can clearly see the significant underlying differences in these different stages and how the $\mathrm{CMC}$ combustion model captures these mechanisms.

\section{Conclusions}

The spark ignition and flame propagation in a turbulent methane jet in ambient air has been simulated using the LES/CMC model with detailed chemistry. Three differernt ignition locations were considered, and the emphasis was on flame kernel formation, flame edge propagation and stabilization. The current LES/CMC predicts these three stages reasonably well. For the flame kernel formation, it is found that the convection dominates the propagation of its downstream edge. The initial downstream and radial flame propagation is discussed through comparison between the OH-PLIF images and the computed expansion extent, showing a good agreement with the expriments. Additionally, the flame first propagates downstream and then upstream towards the nozzle along the other side of the stoichiometric iso-surface. At the flame leading edge, the magnitude of the conditional chemical source term is higher than others, indicating its importance for the flame propagation. The time evolutions of the flame edge position from two ignition cases are compared with measurements and generally good agreement is obtained. At stabilization point, the conditional flame strucures at the leading edge reach the balance between chemistry and micro-mixing. This is the first investigation where the accuracy of LES/CMC to capture flame propagation across a wide range of flame regimes is validated through quantitative comparisons with the expeimental measurments.

\section{Acknowledgement}

This work used the UK Cirrus Cluster (http://www.cirrus.ac.uk/). HZ thanks the financial support from National University of Singapore (R-265-000-604-133). 


\section{References}

[1] E. Mastorakos, Prog. Energy Combust. Sci. 35(2009)57-97.

[2] E. Mastorakos, Proc. Combust. Inst. 36(2017)2367-2383.

[3] J.L. McCraw, N.J. Moore, K.M. Lyons, Flow, Turbul. Combust. 79(2007)83-97.

[4] S.F. Ahmed, E. Mastorakos, Combust. Flame 146(2006)215-231.

[5] N. Chakraborty, E. Mastorakos, Phys. Fluids 18(2006)105103-105120.

[6] A.D. Birch, D.R. Brown, M.G. Dodson, J.R. Thomas, Proc. Combust. Inst. 17(1979)307-314.

[7] A.D. Birch, D.R. Brown DR, M.G. Dodson, Proc. Combust. Inst. 18(1981)1775-1780.

[8] E.S. Richardson, Ignition Modelling for Turbulent Non-premixed Flows, PhD Thesis, University of Cambridge, Cambridge, UK, 2007.

[9] Z. Chen, N. Swaminathan, S. Ruan, Proc. Combust. Inst. 36(2017)1645-1652.

[10] W.P. Jones, V.N. Prasad, Proc. Combust. Inst. 33(2011)1355-1363.

[11] G. Lacaze, E. Richardson, T. Poinsot, Combust. Flame 156(2009)1993-2009.

[12] A. Triantafyllidis, E. Mastorakos, R.L.G.M. Eggels, Combust. Flame 156(2009)2328-2345.

[13] S.F. Ahmed, R. Balachandran, T. Marchione, E. Mastorakos, Combust. Flame 151(2007)366-385.

[14] A. Rosiak, A. Tyliszczak, Int. J. Hydrogen Energy, 41 (2016) 9705-9717.

[15] I.S. Kim, E. Mastorakos, Proc. Combust. Inst., 30 (2005) 911-918.

[16] S. Navarro-Martinez, A. Kronenburg, Proc. Combust. Inst., 32 (2009) 1509-1516.

[17] C.D. Pierce, P. Moin, Phys. Fluids 10(1998)3041-3044.

[18] C. Pera, J. Réveillon, L. Vervisch, P. Domingo, Combust. Flame 146(2006)635-648.

[19] A. Garmory, E. Mastorakos, Proc. Combust. Inst. 33(2011)1673-1680.

[20] A. Garmory, E. Mastorakos, Proc. Combust. Inst. 35(2015)1207-1214.

[21] H. Zhang, A. Garmory, D.E. Cavaliere, E. Mastorakos, Proc. Combust. Inst. 35(2015)1167-1174.

[22] H. Pitsch, H. Steiner, Phys. Fluids 12(2000)2541-2554.

[23] E.E. O’Brien, T. Jiang, Phys. Fluids A Fluid. Dyn. 3(1991)3121-3123.

[24] N. Kornev, E. Hassel, Commun. Numer. Methods. Eng. 23(2007)35-43.

[25] H.G. Weller, G. Tabor, H. Jasak, C. Fureby, Comput. Phys. 12(1998)620-631.

[26] H. Zhang, E. Mastorakos, Proc. Combust. Inst. 36(2017) 1669-1676.

[27] H. Zhang, E. Mastorakos, Flow, Turbul. Combust. 96(2016)863-889.

[28] P.N. Brown, A.C. Hindmarsh, Appl. Math. Comput. 31(1989)40-91.

[29] C.J. Sung, C.K. Law, J.-Y. Chen, Proc. Combust. Inst. 27(1998)295-304.

[30] C.D. Richards, W.M. Pitts, J. Fluid. Mech. 254(1993) 417-435.

[31] M.T.E. Smith, A.D. Birch, D.R. Brown, M. Fairweather, Proc. Combust. Inst. 21(1988)1403-1408. 